\title{
Integrating health and social care assessment and care management: findings from a pilot project evaluation
}

Angela Christiansen Faculty of Health, Edgehill Aintree Campus, Liverpool, UK and Kathryn Roberts Cheshire and Merseyside Strategic Health Authority, Stockton Heath, UK

The implementation of single assessment for vulnerable older people by 2004 was introduced in the National Health Services (NHS) Plan and the National Service Framework for Older People. The evaluation reported and discussed here is of a single assessment and integrated care process (SAP, ICP) piloted and subsequently introduced into a NorthWest of England Primary Care Trust. The initiative introduced enabled district nurses to undertake social assessments and fulfil the role of care manager for people already on their caseloads whose social needs increased.

The objectives of the evaluation were to explore the impact of the initiative on service users and on district nurses and social workers professional roles compared to the previous system of separate assessments and to examine any barriers to the implementation of single assessment and integrated care.

The perceptions of health and social care professionals and service users were collected via telephone and face-to-face semi-structured interviews $(n=21)$.

All professionals could identify both actual and potential benefits and disadvantages to service users of single assessment and integrated care planning. The areas important to respondents included a SAP that is, more efficient, without duplication; familiarity with assessor; timeliness of assessment; increased co-ordination and the quality of assessments. Many of these are also discussed in terms of a change in professional roles, mainly that of district nurses. The introduction of the ICP was not as successful as had been anticipated; a number of reasons for this were identified. The requirement to assess clients' financial circumstances was the main conflict reported for district nurses compared to their traditional role. Issues relating to their lack of knowledge and familiarity of the social care sector were also highlighted. The discussion focuses on how these issues could be addressed namely through education and training, and facilitating integrated working.

Key words: care management; district nursing role; older people; single assessment process; social care

\section{Introduction}

Community care policy over the last two decades has highlighted the need for services and professionals from the National Health Services (NHS) and local authority social services departments to work together more effectively (Higgins et al.,

Address for correspondence: Angela Christiansen, Curriculum Adviser, Faculty of Health, Edgehill Aintree Campus, University Hospital Aintree Site, Longmoor Lane, Liverpool, L9 7AL.
1994; Glendinning and Rummery, 1998; Vernon et al., 2002). Kesby (2002) argues that the division between health and social care provision has been one of the most problematic and contentious issues since the NHS was formed and a distinction was made between the 'sick' and the 'frail'.

Recently there has been fresh impetus to bridge the health and social care divide. Fundamental to the reforms of the NHS Plan (DH, 2000) is the requirement for organizational barriers to be broken down so that the needs of patients are put at the 
centre of the care process. Strengthening the commitment to integrate health and social care services, The National Service Framework for Older People introduced the notion of a single assessment process (SAP) which brings together health and social care assessment, commissioning and provision of services and equipment (DH, 2001). It is anticipated that a single assessment will ensure that older people receive an appropriate and responsive package of care regardless of health and social boundaries. It has been heralded as an opportunity to streamline the assessment process, reduce duplication and inconsistency of assessment and to ensure assessment is more client centred and holistic.

It is well recognized that poor co-ordination between health and social care services can have a devastating effect on vulnerable older people. The Department of Health White paper, Modernising Social Services (DH, 1998), is complementary to The New NHS, Modern and Dependable (DH, 1997) and details concerns about ineffective care at the health and social care interface.

It is recognized that older people often have complex multidimensional needs that span the spectrum of social care, primary care and secondary care. Guidance on implementing the single assessment (DH, 2002) suggests that a range of professionals including district nurses and social workers, using a common assessment tool, can undertake a full assessment. Indeed it is implied that these two professional groups share an interface of concern and expertise.

Worth (2001) argues that assessment of need at the level of the individual is the process underpinning all other aspects of integrated community care. However, research exploring health and social assessment practices are somewhat limited and are hampered by the lack of agreement about what represents a good assessment, both within and between professional groups (Nolan and Caldock, 1996).

Parry-Jones and Soulsby (2001) used a qualitative design to explore the changing assessment practices of health and social care professionals to identify the extent to which assessment was needs led. The study found a lack of consensus about the objectives of assessment both within and across disciplines and lack of a coherent framework for undertaking needs assessment. Practitioners indicated that, whilst they supported the idea of needs-led care, implementing this in practice was difficult, mostly due to resource constraints.
Using an ethnographic approach, Worth (2001) explored the different assessment practice of social workers and district nurses. Both social workers and district nurses believe their own assessment practice to be holistic; however, Worth (2001) observed that social workers focussed on the social factors affecting an individual, whilst nurses concentrated on the impact of the medical condition on an individuals' ability to care for themselves. It is suggested that although each professional group claimed cross boundary expertise the reality seldom supported this assertion.

It is suggested that the assessment process of the two professional groups is heavily influenced by professional education, organizational and resource factors (Worth, 2001). This is supported by Vernon et al. (2000) who suggests that for social workers, assessment involves identifying individual eligibility for services and subsequent allocation of resources, whilst within health care, assessment is used to facilitate diagnosis and plan appropriate nursing and medical interventions and may include referral to social services. This differing orientation may hamper effective collaborative assessment.

\section{The single assessment and integrated care pilot}

The integrated care pilot (ICP) was introduced to three general practitioner (GP) practices in a NorthWest of England Primary Care Trust in April 2000. From April 2001, a further two GP practices were included. Essentially the ICP involved the introduction of a SAP enabling district nurses to undertake social assessments and fulfil the role of care manager for people already on their caseloads whose social needs increased. Similarly, GPs in these practices, who identified an increase in social needs, could refer on to a district nurse to undertake the assessment and care planning. District nurses then discuss the outcome of the assessment with the Social Services Team Manager who can submit a request for funding to the allocation panel in order for the services to be provided.

The previous system, and indeed the system for those practices not involved in the ICP, was that district nurses or GPs who identified an increase in social needs would make a referral to the Social Services Customer Services unit for a social worker to be allocated. During this time, and until 
alternative services are put in place, the district nurse continues to visit. It was anticipated that introduction of a single assessment would result in less duplication, more timely interventions, improved working between health and social services and improved quality of assessment.

Initial funding for the ICP came from the Joint Investment Plan for Older People with the aim of introducing a multidisciplinary assessment tool to bring health and social services closer together. At this time funding was provided jointly by the local authority Social Services and the Primary Care Trust. The process of developing a multidisciplinary tool involved wide consultation with all relevant disciplines and was ongoing alongside the implementation and evaluation of the ICP. The single assessment document incorporates all assessments that may be undertaken and is for use by all professionals.

Prior to implementation, awareness-raising sessions were held for health and social services staff involved in the ICP. Two half-day training sessions were also held for the $\mathrm{G}$ and $\mathrm{E}$ grade nurses from the three pilot practices. The half-day training sessions covered care assessment and care management, documentation, eligibility criteria, the panel process, the process of commissioning services and the review process.

To support the implementation of the SAP, a number of new posts were established. These included a part-time finance and administration officer to undertake the financial aspect of assessment and a nurse 'champion' to provide support, guidance and advice to district nurses undertaking assessments. In addition bank staff were recruited to replace nursing hours devoted to assessment. The continuing support and advice to district nurses from social workers, social service managers and the integrated assessment co-ordinator were also available.

This paper reports findings from an evaluation study of the SAP introduced as part of an ICP. Data were collected between April 2001 and April 2002.

\section{Methodology}

The objectives of the study were:

- To understand stakeholders perceptions of the benefits and disadvantages that a single health and social care assessment had brought for service users and their carers.
- To examine the perceived impact of ICP on the role and practice of district nurses and social workers.

- To identify barriers to full implementation of the SAP and examine how these could be overcome, as perceived by all major stakeholders.

An evaluation approach was undertaken to explore the development and implementation of the ICP. Evaluation research recognizes the contribution of major stakeholders in the evaluation process. Patton (1990) defines stakeholders as people who have a vested interest in the evaluation findings. Within this study, the stakeholders were identified as health and social care professionals, service users and informal carers.

The key evaluation questions and the tools used to effectively address them were determined and designed during the planning stage of the study and discussed with individuals with key roles in the design and implementation of the ICP. The local research ethics committee granted ethical approval for the study.

Data were collected using semi-structured interviews conducted with 21 professionals. A qualitative approach allowed greater depth of responses to be gained, facilitating deeper understanding of the process under investigation (Patton, 1990; Marshall and Rossman, 1995).

Health and social care professionals were purposively sampled for inclusion in this study. That is, posts were identified, the holders of which were contacted as potential interviewees (Table 1). Interviews with professionals were conducted over the telephone or in the workplace of the respondent. Interviews were tape recorded with the respondent's consent.

Table 1 The Research Participants

Team Manager, Social Services

Area Manager, Social Services

Clinical Services Manager, Primary Care Trust

Clinical Development Co-ordinator

Integrated Assessment Co-ordinator

Finance and Administration Officer, Social Services

National Service Framework for Older People Lead, Health Authority

Five Social Workers

Five District Nurses

Four Service Users 
Transcribed data from interviews were subjected to thematic analysis (Denzin and Lincoln, 1994; Silverman, 2001). Interview transcripts were comprehensively grouped and labelled to determine the areas important to respondents. The interview guides and the evaluation questions structured much of the preliminary analysis.

Computer-aided content searching was also carried out to supplement manual coding. The qualitative analysis package Nud Ist (version 4.0) was used to assist in organizing and managing the data.

\section{Findings}

\section{Consequences of the single assessment for service users}

District nurses, social workers and service managers could identify both actual and potential benefits and drawbacks to service users of a comprehensive single assessment undertaken by a district nurse.

\section{One assessment}

The benefit cited by most interviewees was that service users would not have to give the same information to different professionals, which represents both duplication and inefficiency and is irritating and an intrusion for the service user and informal carer.

This was expressed in the following ways: 'less people coming in', 'people aren't having to give the same information time and time again' and 'eradicate duplications and overlapping'. It was suggested by one participant that an older adult who had been admitted and subsequently discharged from hospital could have up to 12 assessments in one year all asking the same questions.

\section{Familiarity with assessor}

Many participants suggested, as clients already have a relationship with the district nurse, trust had been established and service users may be more at ease providing new information. Indeed many participants suggested that district nurses were in an ideal position to undertake this role, given their long-term relationship with the people they assess:

The district nurse has got a good relationship with the carer and relatives so they have the whole knowledge of that person.

(District Nurse)
Service users confirmed an established relationship with the district nurses:

When my husband came home (from hospital) the $\mathrm{d} / \mathrm{n}$ 's came in every other morning to dress his leg. So we were quite used to them all coming. Yes, you see she (district nurse) came right from the start ... the $\mathrm{d} / \mathrm{n}$ was one of the first to come ... they pop in now if they are passing, we've got very friendly.

(Service User)

\section{One point of contact}

It is often argued that people with both health and social needs have difficulty distinguishing between their needs in any way that corresponds to present boundaries between health and social care service provision (Twigg, 1997, Rummery, 1998). Some respondents commented upon the consequences of such demarcation on service users, for example:

(Saying to Service Users) We provide this bit but we don't provide that bit, that is the health department that provides the commode ... people find that very annoying and upsetting and very stressful.

(Social Worker)

Having a named district nurse to contact with any care needs or questions was seen to have the potential to reduce the confusion that older people feel when faced with the complex array of services and different providers. This confusion was evident from service users. When asked if she had seen a social worker one service user responded:

No, I get mixed up with social services, something and something, there's several, all these different people, but I don't think I have seen one, the District Nurses have done everything for me.

(Service User)

\section{Timeliness of assessment and service provision}

A factor in the rationale for the introduction of the ICP was that assessments would be more timely and reactive as district nurses could detect and respond immediately to increased social need as it arose. Service users are likely to receive an assessment sooner by a district nurse, who can undertake it immediately, rather than waiting 
to be allocated a social worker. The following comment demonstrates the potential for timely provision:

They can see that there is a need, then they can deal with it without having to refer to somebody else. It can make things happen much more quickly.

\section{(Clinical Services Manager)}

While high-priority cases referred to Social Services are responded to within agreed time scales, professionals who had made referrals to Social Services via the Customer Services line suggested it could take 10-14 days or longer before a social worker undertook an assessment. It was envisaged that service users should experience less waiting for both assessment and care provision. Indeed, one district nurse noted that within two hours of a client assessment, services had been commissioned and those services went in the same day. Enhanced speed of implementation of services was seen as essential to prevent further deterioration and avoid crises.

One participant however questioned the equity of a social assessment that could result in quicker care provision:

Perhaps (district nurses) would see it as a way of short cutting access to our system. Whether that is an equitable way ... if you have got a district nurse you can short cut the system, otherwise you wait for your priority.

(Social Worker)

\section{Increased co-ordination}

The facilitation of a continuous and co-ordinated process, from recognition of need, through assessment to care provision was the remaining major advantage to service users and informal carers cited by professionals. This again stems from the advantage that one person assumes responsibility for carrying out an holistic assessment and arranging a package of care and acts as a contact point for the service user. It was thought that service users may be advantaged as district nurses could keep them informed of the progress of their application for care and the likely waiting times; for example:

They are in a position where they are not only able to do the assessment but to give some indication to the service user of where it will go from there.

(Integrated Assessment

Co-ordinator)

\section{Quality of assessment and care planning}

The main source of disadvantage or potential disadvantage identified by all but four professional interviewees was the relative lack of experience of district nurses in undertaking social assessments and their relative lack of knowledge of the social care sector. This had two components, first that nurses did not have the knowledge and skill to collect the right information and secondly that district nurses are less knowledgeable of services available and eligibility criteria. Examples were given of district nurses not completing the right paperwork or not distributing completed paperwork to the correct people. Some of the ways in which the district nurses relative lack of knowledge of Social Services may be disadvantageous to service users were expressed thus:

(District Nurses) perhaps don't know how social care fits into the wider context in terms of accessing voluntary groups, a more holistic picture of social care.

(Social Worker)

Sometimes it could be a disadvantage to the user in as much as we don't know what is available to them.

(District Nurse)

No indication was given that these difficulties would continue into the long term as district nurses gained more knowledge and experience.

\section{Impact upon the role of the district nurse}

The district nurses are the professional group central to the implementation of the ICP and therefore the group upon whom it could be expected to have the greatest impact. All professional interviewees were asked for their experience and perceptions of the positive and negative ways in ICP had impacted on the role of the district nurse.

\section{Autonomy and expanding expertise}

The positive impact, mentioned most frequently, which had resulted or could result from 
the ICP upon the professional role of district nurses was that they had more 'autonomy' over the provision of care and were able to oversee the assessment, progress and management of care provision:

(when) I was a district nurse ... you always felt like your hands were tied because somebody else had to come and finish off what you had started.

\section{(Clinical Development Co-ordinator)}

A second advantage of the ICP for district nurses was that district nurses could expand their expertise by becoming more knowledgeable of the social care sector. Some of the respondents commentated upon the potential for mutual learning:

It is bringing (district nurses and social workers) together more and we are each understanding roles, they are perhaps understanding more of our role.

(Social Worker)

\section{Workload}

It was acknowledged by many participants that incorporating the single assessment into the role of the district nurses presented them with many challenges. The one negative aspect discussed by all professionals was the impact upon the workload of district nurses. All professionals perceived that it meant more work for district nurses, namely that it was an increased workload, that it was time consuming and the paperwork involved.

Increased workload was discussed by district nurses as a 'major disadvantage', an 'extra burden' and 'adding that responsibility onto district nurses who are already seeing to the person's physical well-being'.

The paperwork involved was discussed as both an extra role for district nurses to fulfil but also as being unfamiliar and complex:

The staff are just overwhelmed with the number of forms that they have to complete for social services ... I think that has been crucial in the non-success of the scheme ... if it is going to take them half a day to do all the paperwork and then all the follow up paperwork.

(Director of Partnerships and Modernisation)

\section{Financial assessments}

In addition, a fundamental aspect of assessment for social care undertaken by social workers is establishment of clients' financial circumstances as many services are means tested and can incur a charge. The requirement to do such assessments was discussed by many interviewees as a negative way in which the ICP could impact upon the role of district nurses:

I don't think some of the district nurses are comfortable in talking about finances because the service they give is free at the point of delivery and we have quite strict eligibility criteria and strict financial controls.

\section{(Team Manager, Social Services)}

It was suggested that district nurses were finding ways of circumventing this requirement, i.e., they were referring service users to either the Rapid Response Service or Palliative Care Service, as these had the ability to increase care provision without financial cost to the individual:

It was noticeable early on of course that the nurses were far keener to do assessments which lead to a day centre application because there is no financial assessment for day care, than say for a care package which there is.

(Social Worker)

Many social workers anticipated that nurses would find financial assessment challenging as they also had concerns about undertaking this aspect of assessment:

We have to do the dual role of finance officer and care manager and assessor. We felt (District Nurses) were lucky getting a finance person, there should be someone within Social Services or any assessment procedure a separate finance officer because it does detract from the assessment and from the relationship really ... It is an extremely complex part and you have to do the two in the one visit, it does go against the care management process.

(Social Worker)

\section{Impact upon social services professionals}

A fully implemented system of integrated care, based on the pilot, would mean fewer referrals to Social Services for assessments and care 
management. However due to the low throughput to the ICP and due to the nature of a pilot scheme Social Services professionals also had an additional role to 'buddy' district nurses and provide advice and support. All professionals were asked for their perceptions of the positive and negative aspects of the ICP to the role of social workers.

\section{Fewer referrals}

The two main positive factors cited were fewer referrals being made to social workers and the potential for increasing their knowledge of the health care sector. Fewer referrals for social care assessment can be viewed both as a positive and as a negative change and interviewees were asked to expand upon their contradictory viewpoints. At one level social workers felt that the large number people eligible for single assessments to be undertaken by district nurses had little impact upon their overall assessment role:

It saves us an afternoon of paperwork ... the amount of care packages that pass through our hands compared to what they are able to pick up ... they were picking up on ones that would have come through as priority one.

(Social Worker)

\section{Threat to the role of social workers}

The major negative way in which the ICP may impact upon the role of social workers was that it may be perceived as a 'threat' to their role or an 'erosion' of their role as well as a devaluation of their skills or an 'undermining of their abilities' both at an individual level and for the whole profession. That is compounded as district nurses increase their role in social care without any reciprocal increase in the role of social workers in the health care sector. Many social workers felt uncertain about their future role:

You could say that perhaps we would be out of a job, they are doing both then what is the need for us ... I really don't know where we are going and what we will be doing.

(Social Worker)

\section{Barriers to implementation}

Many interviewees identified the different cultures of health and social care as a factor causing the implementation of the ICP to be problematic. Some of the comments demonstrate how this 'cultural' difference may explain some of the ways in which assessments undertaken by district nurses differ from those undertaken by social workers:

In our service, we do the assessment without any view to the care plan that will be set up after, but we have a very strict criteria for getting an assessment in the first place ... We are much more gate keeper lead for assessment ... We only focus on the management of care. They are nursing as well. It is a dual role and I don't think it will sit well really.

(Social Worker)

In addition some social service participants suggested that district nurses might find familiarity with the service user may compromise the assessment process as district nurses might find it difficult to inform of an outcome, which does not support the service users wishes. It is suggested by Vernon et al. (2002) that the assessment process within local authorities acts as a form of rationing and is not always an accurate reflection of the needs of the service user. Indeed Worth (2001) notes how resource constraints present ethical dilemmas for social workers who identify need that cannot be met.

\section{Overcoming barriers}

During the interviews with professionals, 'closer integration' of district nurses and social workers was identified as a way of taking forward single assessment and care and moving towards 'seamless care' for the benefit of service users. Closer integration was conceptualized on a number of levels, including an individual level, group level, systems level and education and training.

\section{Closer integration at an individual level}

For some respondents the desired outcome of closer integration involved achieving closer geographical proximity. There was some consensus among health staff that a shared geographical base and the daily interaction this would make possible would facilitate better joint working:

To see each other and talk to each other ... discuss things more ... bounce ideas off each other.

(District Nurse) 
Integration at a group level: working in teams

Other respondents elaborated upon this by suggesting that closer integration required not just common location but a common purpose to more closely align the different 'cultures' of health and social care.

Many participants believed that fully integrated teams would assist in achieving the right skill mix and help facilitate understanding of other professional roles. As well as district nurses and social workers, other suggested professionals include administrative staff, financial administrators, health visitors, consultants, GPs, occupational therapists, physiotherapists, counselling services, support workers, auxiliary nurses, practice nurses and links to voluntary organizations:

If you had a team, a particular patch team that was working and meeting together, then you would be much more aware of how things were going but if you are always doing it on the end of the phone or somebody has a referral sent to them, inevitably things aren't followed up the same way.

(Social Worker)

Many participants had experience of the interdisciplinary Rapid Response Service, in which nurses, a physiotherapist and a social workers pooled expertise to meet the needs of acutely ill people in their own homes. This was seen by district nurses as a positive example of effective integrated care.

\section{Shared systems, management structures and resources}

Suggestions for other developments to facilitate fully integrated teams were for NHS and Social Services systems and processes to become more closely aligned. This included shared records, shared eligibility criteria, 'pooled budgets', 'a single IT system' and shared administration.

Sharing information or having the ability to 'access each other's records and files' was identified by a number of individuals with experience of difficulties caused by separate records. One district nurse discussed how when visiting patients she was not always aware if there had been Social Services involvement and one social worker identified a situation when they had started an assessment and the service user alerted them that a district nurse had previously been and carried out the same assessment. Many participants felt that access to records across disciplines would help eradicate such uncertainty, increase efficiency and improve patient care:

Shared information bases, records, IT ... you should be able to key into their records to check when they were last assessed, who that person was, what the care plan was.

(Social Worker)

We are working two systems and although you can get individuals to work together, the systems are incompatible.

(District Nurse)

Many participants particularly at service planning level had awareness of the need to move towards more integrated planning and management of services to cross the health and social care divide. While the difficulties in achieving this were acknowledged, many regarded this an inevitable process. There was general consensus that this would be in the best interests of service users and carers with complex needs:

Joint management would make a much better strategy. Health and Social Services are inevitably going to have different management pulling in different ways ... You are managing two different pots of money, two lots of bosses trying to do things their way.

(Social Worker)

\section{Education and support}

Most respondents acknowledged that further education and training would be beneficial. District nurses in particular felt that initial training had been minimal and not adequate for such a significant change in working practices. In particular nurses wanted training that had a practical focus including the acquisition of skills relating to assessing social needs, to use documentation and eligibility criteria, how to ask about finances as well as experience of the allocation panel.

Many supported the idea of development that was practically orientated involving district nurses and social workers working together on real case examples. Strategies were identified to support this, such as district nurses secondment onto social worker teams for three months, as with social 
worker students. Professionals from both district nursing and social work suggested that shadowing of each other would enable greater understanding of each other's roles and allow social workers to gain more knowledge of health assessments.

\section{Conclusion}

Findings from this evaluation suggest that there are benefits of single assessment and integrated care management for the service user and their carer. The assessment takes place as increased need is detected and can mean more timely, reactive and co-ordinated implementation of services and may prevent further deterioration and crisis. The service user is only required to provide the assessment information once and the assessment event is undertaken within an established relationship, with a professional who is already known to the client and family. The perceived disadvantages to service users are associated with district nurses' limited knowledge and familiarity with the social care sector.

While all participants acknowledged that the SAP had positive benefits for the service users, many district nurses expressed concern over the time taken to complete the assessment forms and lack of confidence and knowledge of social service provision. In addition district nurses experienced role conflict in undertaking financial assessment. To nurses, assessment is a method of gathering information to enable care to be planned, implemented and evaluated whereas for social care, it is a commissioning task to identify eligibility for services and allocation of resources.

The SAP is a key element of the National Framework for Older People and NHS bodies and social services were required to achieve full implementation by 2004. The ICP is an innovative attempt to go some way to achieving this target. Initial evaluation data would suggest that most professional involved agree that it has the potential to ensure that service provision is more person centred and can promote continuity of care across the health and social care interface.

The success of such initiatives may depend on overcoming barriers to the implementation of integrated care. This will depend on adequate support for the professions involved, and continuing education and training to bring health and social care providers into closer alignment so that policy objectives are delivered to clients and their carers.

\section{References}

Department of Health. 1990: The National Health Service and Community Care Act. London: Her Majesty's Stationery Office.

Department of Health. 1997: The New NHS Modern Dependable. London: The Stationery Office.

Department of Health. 1998: Modernising Social Services. London: Department of Health.

Department of Health. 2000: The NHS Plan. A Plan for Investment, a Plan for Reform. London: Department of Health.

Department of Health. 2001: National Service Framework for Older People. London: The Stationery Office.

Department of Health. 2002: The Single Assessment Process: Guidance for Local Implementation. London: The Stationery Office.

Denzin, N.K. and Lincoln, Y.S. 1994: Handbook of Qualitative Research. Thousand Oaks, CA: Sage.

Glendinning, C. and Rummery, K. 1998: A duty of partnership: bringing health and social care together. British Journal of Health Care Management 4, 294-97.

Higgins, R., Oldham, C. and Hunter, D.J. 1994: Working together: lessons for collaboration between health and social services. Health and Social Care 2, 269-77.

Kesby, S. 2002: Nursing care and collaborative practice. Journal of Clinical_Nursing 11,357-66.

Marshall, C. and Rossman, G.B. 1995: Designing Qualitative Research, second edition. London: Sage.

Nolan, M. and Caldock, K. 1996: Assessment: identifying the barriers to good practice. Health and Social Care in the Community 4, 77-85.

Parry-Jones, B. and Soulsby, J. 2001: Needs-led assessment: the challenges and the reality. Health and Social Care in the Community 9, 414-28.

Patton, M.Q. 1990: Qualitative Evaluation and Research Methods, second edition. Newbury Park, CA: Sage.

Rummery, K. 1998: Changes in primary health care policy: the implications for joint commissioning with social services. Health and Social Care in the Community, 6, 429-37.

Silverman, D. 2001: Interpreting Qualitative Data. Methods for analysing talk, text and Interaction. London: Sage.

Twigg, J. 1997: Deconstructing the 'social bath': help with bathing at home for older and disabled people. Journal of Social Policy 26, 211-32.

Vernon, S., Ross, F. and Gould, M. 2000: Assessment of older people: politics and practice in primary care. Journal of Advanced Nursing 31, 282-87.

Worth, A. 2001: Assessment of the needs of older people by district nurses and social workers; a changing culture? Journal of Interprofessional Care 15, 257-66. 